\title{
Lipopolysaccharide induces expression of collagen VI in the rat lung
}

\author{
Sayuri Okawa ${ }^{1 \#}$, Kana Unuma ${ }^{1 \# *}$, Atsushi Yamada ${ }^{1}$, Toshihiko Aki ${ }^{1}$, and Koichi Uemura ${ }^{1}$ \\ ${ }^{1}$ Department of Forensic Medicine, Graduate School of Medical and Dental Sciences, Tokyo Medical and Dental University, \\ 1-5-45 Yushima, Bunkyo-ku, Tokyo 113-8519, Japan
}

\begin{abstract}
The involvement of the lung during the septic systemic inflammatory response elicited by administration of lipopolysaccharide (LPS) was investigated. Eight-week-old male Sprague-Dawley rats were injected i.p. with $15 \mathrm{mg} / \mathrm{kg}$ LPS. After $24 \mathrm{~h}$, the lungs were excised to evaluate the cellular responses to LPS. Matrix-assisted laser desorption ionization time-of-flight mass spectrometry (MALDI-TOF) analysis revealed that type VI collagen (ColVI) was extremely upregulated during sepsis in the rat lung within the first $24 \mathrm{~h}$ of LPS administration. Upregulation of ColVI protein and its mRNA was demonstrated by Western blot analysis, real time PCR, and immunohistochemistry. To the best of our knowledge, this is the first report demonstrating the activation of ColVI in the rat lung at the early stage of systemic inflammation. Activation of ColVI might be involved in sepsis-mediated lung fibrosis at an early stage. (DOI: 10.1293/tox.2014-0053; J Toxicol Pathol 2015; 28: 37-41)
\end{abstract}

Key words: LPS, lung, type VI collagen, rat

Lung inflammation during sepsis leads to acute lung injury (ALI) and acute respiratory distress syndrome (ARDS), which may trigger persistent lung fibrosis. ALI/ ARDS is manifested by acute severe respiratory failure accompanied by a high mortality rate, and is induced by pulmonary edema due to infection, trauma, or allergy. In the late phase of ALI/ARDS, remodeling of the extracellular matrix (ECM) results in accumulation of connective tissues, mainly composed of collagen, and over-activation of this remodeling leads to mortal lung fibrosis ${ }^{1}$. Although the regulatory mechanisms are not well understood, some proteins associated with regulation of acute lung injury have been proposed, including NF-kappaB, neutrophil elastase ${ }^{2}$, vitronectin ${ }^{3}$, and collagens.

The ECM of connective tissues is comprised of a variety of proteins that define structural integrity and various physiological functions. The most abundant family is the collagens comprising $>20$ different types identified to date. Collagens are centrally involved in the formation of fibrillar and microfibrillar networks of the ECM, such as basement membranes as well as other structures of the connective tissues $^{4}$. In the lung epithelium, the basement membrane contains fibronectin, laminin, and collagen VI (ColVI) ${ }^{5}$. ColVI is a widely distributed ECM protein forming microfibrillar

Received: 5 November 2014, Accepted: 2 December 2014

Published online in J-STAGE: 27 December 2014

*Corresponding author: K Unuma (e-mail: unuma.legm@tmd.ac.jp)

\# These authors contributed equally to this study.

(C)2015 The Japanese Society of Toxicologic Pathology

This is an open-access article distributed under the terms of the Creative Commons Attribution Non-Commercial No Derivatives (by-ncnd) License $<$ http://creativecommons.org/licenses/by-nc-nd/3.0/>. networks with proteoglycans as well as other collagen fibers, and is involved in formation of the basement membrane $^{6-8}$. The triple helix of ColVI is typically composed of alpha1, alpha 2 and alpha 3 chains and is secreted into the ECM after forming a tetrametric structure?

In humans and other vertebrates, ColVI is present in the vascular and bronchial walls and in the interstitial space in the lungs ${ }^{10}$. In addition, at the early stage of lung fibrosis, ColVI expression is increased and increased mRNA expression is mainly observed in fibroblasts of the myofibroblast type $^{11}$. Recently, we showed that administering $15 \mathrm{mg} / \mathrm{kg}$ lipopolysaccharide (LPS) to rats for $24 \mathrm{~h}$ induced systemic inflammation and activated lysosome biogenesis and autophagy in the liver and heart ${ }^{12,13}$. Here we found that systemic inflammation activates ColVI in the rat lung, as an early phase of fibrosis.

The animal experiment protocols used in this study were approved by the Institutional Animal Care and Use Committee of the Tokyo Medical and Dental University. In brief, eight-week-old male Sprague-Dawley rats were injected i.p. with $15 \mathrm{mg} / \mathrm{kg}$ LPS (from Escherichia coli obtained from Sigma [L-2630; St Louis, MO, USA], 500,000 Endotoxin Units/mg) dissolved in $0.5 \mathrm{~mL}$ isotonic $\mathrm{NaCl}$ or vehicle ( $n=4$ / group). LPS was injected i.p. into the rats 24 $\mathrm{h}$ prior to sacrifice.

Lungs $(0.1 \mathrm{~g})$ were homogenized with $1 \mathrm{~mL}$ of ice-cold STE buffer containing $320 \mathrm{mmol} / \mathrm{L}$ sucrose, $10 \mathrm{mmol} / \mathrm{L}$ Tris-HCl (pH 7.4), $1 \mathrm{mmol} / \mathrm{L}$ EDTA, $50 \mathrm{mmol} / \mathrm{L} \mathrm{NaF,} 1$ $\mathrm{mmol} / \mathrm{L} \mathrm{Na}_{3} \mathrm{VO}_{4}$, and Complete Protease Inhibitor Cocktail (Roche Diagnostics, Mannheim, Germany). Samples were subjected to SDS-PAGE using a 7.5\% gel. For MALDI-TOF analysis, proteins were resolved by SDS-PAGE and several proteins were excised, reduced, S-alkylated, and digested 
with trypsin (sequence grade modified; Promega, Madison, WI, USA). The obtained tryptic peptides were subjected to a MALDI-TOF analysis (UltrafleXtreme, Bruker Daltonics, Billerica, MA, USA). The spectra obtained were subjected to a search against the NCBI database using the Mascot search engine (Matrix Science). Western blot analysis was performed with anti-ColVI (Abcam; 1:1,000 dilution), antiColI (ab34710, Abcam, Cambridge, MA, USA; 1:1000 dilution), or anti-GAPDH (Millipore) antibodies. Peroxidaseconjugated antirabbit and antimouse $\operatorname{IgG}$ antibodies were obtained from Promega (Madison, WI, USA). Protein levels were determined using a standard curve constructed by plotting the band densities and was normalized to the GAPDH levels using the CS Analyzer v. 3.0 software (ATTO, Tokyo, Japan).

Complementary DNA was synthesized from lung tissues using TRIzol (Invitrogen, Carlsbad, CA, USA) and SuperScript II Reverse Transcriptase (Invitrogen). Quantitative RT-PCR (qPCR) (StepOnePlus, Applied Biosystems, Foster City, CA, USA) was performed using SYBR green as a fluorescent dye. The primers used were as follows: 5'-GTACATCAGCCCAAACCCCA-3' and 5'-CAGGAT-CGG-AAC-CTT-CGC-TT-3' for Coll-alphal; 5'-CCC-TGG-TGG-ACA-AGG-TGA-AA-3' and 5'-CGCATG-AGC-CCT-CTG-ATG-AT-3' for ColVI-alpha1; 5'-TGG-AAG-ACG-TCC-TTT-GTC-CG-3' and 5'-GTAGAA-GTT-CTG-CTC-GCC-CA-3' for ColVI-alpha2; 5'-GGG-ACA-CAC-GTC-TTC-AGG-TT-3' and 5'-CCATGA-CTG-ATT-GTT-GTT-GGG-3' for ColVI-alpha3; and 5'-CAT-CCG-TTC-TCT-ACC-CAG-CC-3' and 5'-AATTCT-GAG-CCC-GGA-GTT-GG-3' for 18S rRNA. The conditions for the PCR reactions were as follows: $95^{\circ} \mathrm{C}$ for 20 $\mathrm{s}$, followed by 40 cycles of $95^{\circ} \mathrm{C}$ for $1 \mathrm{~s}$ and $60^{\circ} \mathrm{C}$ for $20 \mathrm{~s}$.

Lung tissues fixed in paraformaldehyde were embedded in paraffin, sliced in 3- $\mu \mathrm{M}$-thick sections and stained with hematoxylin-eosin (HE). In addition, in order to identify the location of ColVI and myofibroblasts, samples were subjected to immunohistochemical analysis as previously described ${ }^{12}$. In brief, sections were incubated at $4^{\circ} \mathrm{C}$ overnight with 1/100 diluted anti-ColVI antibodies (ab6588, Abcam, Cambridge, MA, USA) and alpha smooth muscle actin ( $\alpha$-SMA) antibodies (A2547, Sigma), and subsequently incubated with peroxidase-conjugated secondary antibody (Nichirei Bioscience, Tokyo, Japan). Diaminobenzidine was used as a substrate to visualize antigens. The samples were eventually analyzed using a light microscope (Olympus AX80).

To evaluate the cellular responses to LPS in the rat lung, we first examined any major changes in the composition of lung proteins using conventional electrophoresis and protein staining with Coomassie Brilliant Blue. A protein with an apparent molecular weight of $108 \mathrm{kDa}$ was clearly upregulated at $24 \mathrm{~h}$ following LPS treatment (Fig. 1a). MALDI-TOF analysis revealed that the protein was type VI collagen (ColVI), a widely distributed ECM protein (Table 1). Western blot analysis confirmed a significant upregulation of ColVI in the LPS-treated rat lung compared with the a

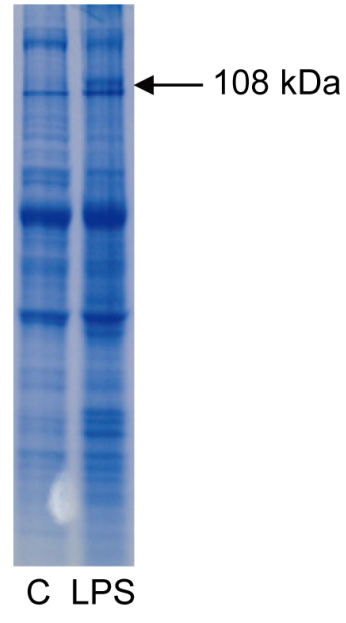

b
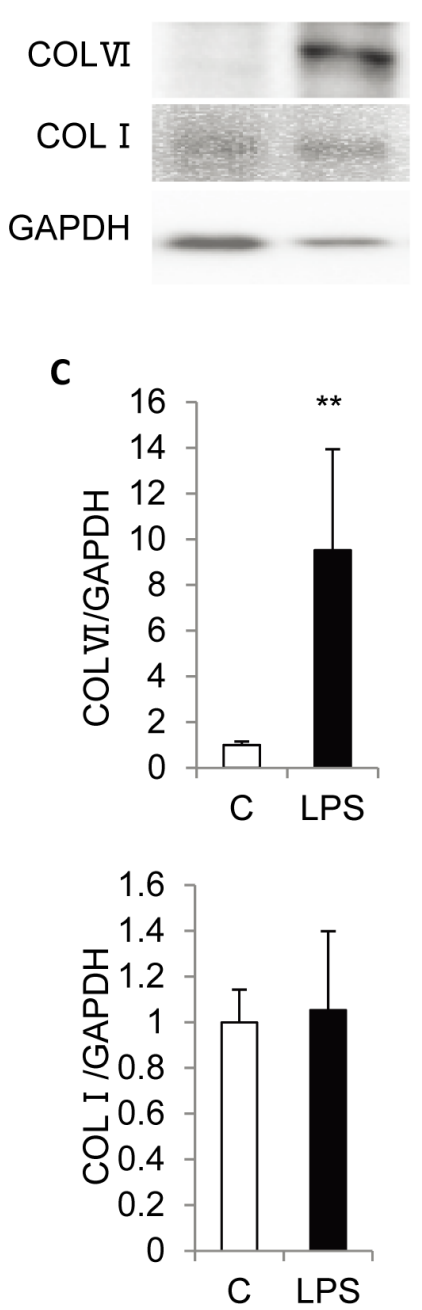

Fig. 1. Increase of ColVI protein in LPS-treated rat lungs. (a) Major changes in the protein composition of the rat lung treated with LPS (15 mg/kg for $24 \mathrm{~h}$ ) as shown by conventional electrophoresis (SDS-PAGE) and protein staining with Coomassie Brilliant Blue. The arrow indicates the major LPS-responsible protein. (b) Western blot analysis of the rat lung treated with LPS $(15 \mathrm{mg} / \mathrm{kg}$ for $24 \mathrm{~h})$ to determine the levels of ColVI and ColI. GAPDH was used as a loading control. (c) Ratios of ColVI, ColI, and GAPDH determined using densitometry analysis. Each bar represents the mean $\pm \mathrm{SE}(\mathrm{n}=4)$. **, $\mathrm{p}<$ 0.01 versus control by Student's t-test. 
Table 1. Tryptic Peptides of the Rat ColVI Observed by MALDI-TOF Analysis

\begin{tabular}{ccccl}
\hline${\text { Observed }(\mathrm{m} / \mathrm{z})^{\mathrm{a}}}^{\mathrm{a}}$ & ${\text { Theoretical }(\mathrm{m} / \mathrm{z})^{\mathrm{b}}}^{\mathrm{E}}$ & ${\text { Error }(\mathrm{ppm})^{\mathrm{c}}}^{\mathrm{s}}$ & Start-end $^{\mathrm{d}}$ & Amino acid sequence $^{\mathrm{e}}$ \\
\hline 943.5153 & 942.4771 & 32.9 & $871-878$ & EDPTQVVR \\
1052.5936 & 1051.5774 & 8.47 & $722-730$ & LLPPTQNNR \\
1073.6187 & 1072.6029 & 8.42 & $1012-1020$ & VPNYQALLR \\
1289.6851 & 1288.6775 & 8.42 & $997-1007$ & TAEYDVAFGER \\
1289.6851 & 1288.6775 & 0.26 & $208-218$ & LSIIATDHTYR \\
1580.8339 & 1579.8358 & -5.84 & $194-207$ & VFSVATPDHLEPR \\
1701.8555 & 1700.8595 & -6.61 & $879-894$ & VAVVQYSGQGQQQPGR \\
1985.8093 & 1984.8190 & -8.51 & $249-264$ & NNVEQVCCTFECQAAR \\
2029.9623 & 2028.9727 & -8.74 & $702-719$ & LQWMAGGTFTGEALQYTR \\
2199.0964 & 2198.1120 & -10.39 & $91-109$ & NLVWNAGALHYSDEVEIIR \\
2713.3218 & 2712.3469 & -11.95 & $36-59$ & AIAFQDCPVDLFFVLDTSESVALR \\
\hline
\end{tabular}

${ }^{a}$ The $\mathrm{m} / \mathrm{z}$ values of observed ions by MALDI-TOF analysis. ${ }^{\mathrm{b}}$ The theoretical $\mathrm{m} / \mathrm{z}$ values of tryptic peptides from rat ColVI. ${ }^{\mathrm{c}}$ The differences of $\mathrm{m} / \mathrm{z}$ between observed and theoretical peptide ions. ${ }^{\mathrm{d}}$ The numbers of start and end positions relative to the first amino acid of rat ColVI. ${ }^{\mathrm{e}}$ Amino acid sequences in rat ColVI.

untreated control, whereas the cytoplasmic protein GAPDH was unaltered (Fig. 1b, c). ColI, one of the major matrix proteins, was unchanged (Fig. 1b, c). Therefore, ColVI may be activated at an early stage in the process of lung fibrosis, prior to activation of ColI.

We next performed qPCR analysis to determine whether or not LPS induces the expression of ColVI genes. As shown in Fig. 2, a 2.3- to 9.9-fold induction of the ColVI genes (ColVI alpha 1-3 chains) was observed $24 \mathrm{~h}$ after LPS administration. In contrast, the ColI alpha 1 gene showed no induction after LPS treatment. A ribosomal RNA (18S rRNA) was used as the internal control.

There were no histological abnormalities and a faint positive stain of ColVI in control lung tissue (Fig. 3a, c). In contrast, lung tissue treated with LPS revealed prominent hemorrhage and thickening of the alveolar walls, and the presence of neutrophils indicating early inflammation (Fig. 3b). In addition, LPS treatment showed a higher number of ColVI-positive lung cells compared with the control (Fig. $3 d)$. In contrast, there was no increase in $\alpha$-SMA-positive cells in the LPS-treated rat lung compared with the untreated rats (Fig. 3e, f), suggesting that the upregulation of ColVI occurs before the increase in myofibroblasts during lung fibrosis. Although previous studies indicated that tumorassociated macrophages induce ColVI and promote tumor inflammation $^{14}$, only the increase in neutrophils in the LPStreated rat lung was observed, suggesting that the influence of macrophages may be negligible.

In this study, we demonstrated upregulation of ColVI protein and mRNA during the systemic inflammatory response in the rat lung within the first $24 \mathrm{~h}$. This result reinforces a previous study evidencing ColVI deposition at the early stage of fibrosis and suggests that ColVI is being highly expressed in the lung from the time of injury until fibrosis becomes obvious ${ }^{11}$. Histologically, ColVI is upregulated mainly in the pulmonary interstitial space as previously described ${ }^{10}$.

ColVI has been reported to induce myofibroblast differentiation in heart tissue, which promotes fibrosis after infarction ${ }^{16}$. However, it is likely that other triggers, such as

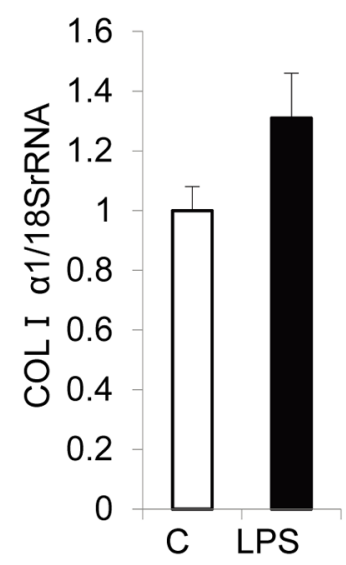

C

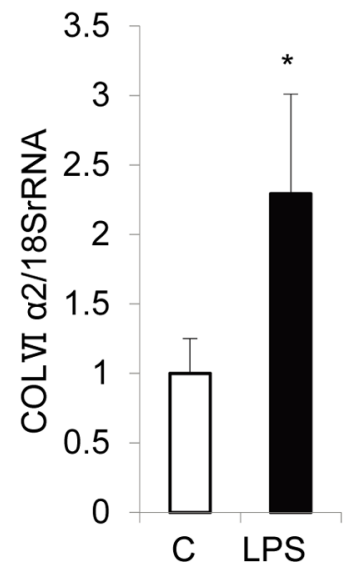

b

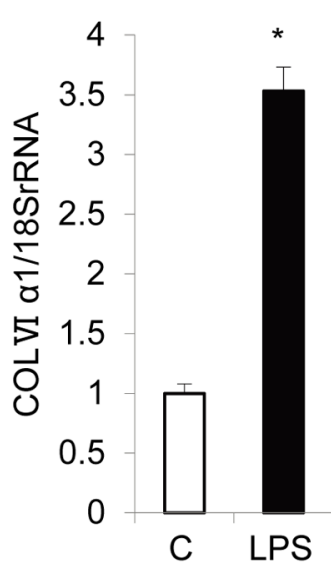

d

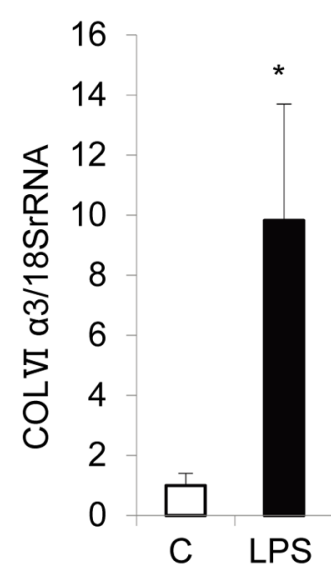

Fig. 2. Activation of ColVI genes. Relative mRNA levels of ColI alpha1 (a), ColVI alpha1 (b), ColVI alpha2 (c), and ColVI alpha3 (d) to GAPDH were analyzed by qPCR. Each graph represents the mean $\pm S E(n=4) . * *, p<0.05$ versus control by Student's $t$-test. 


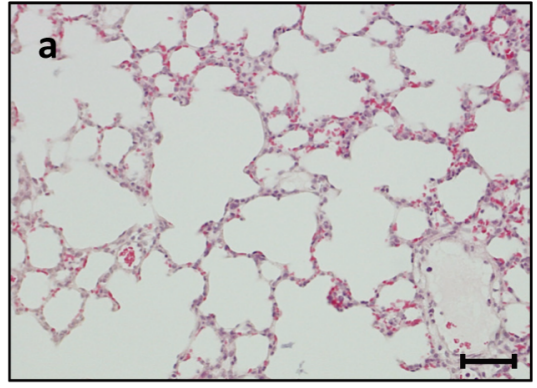

C

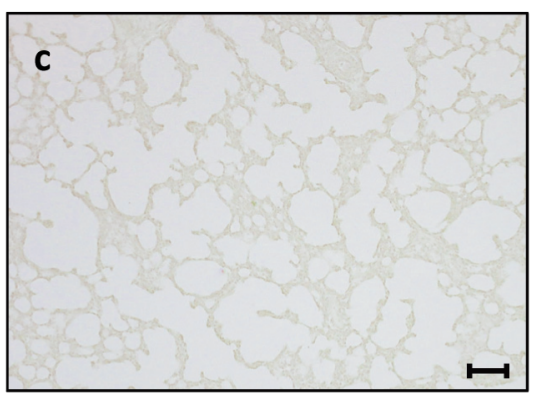

C

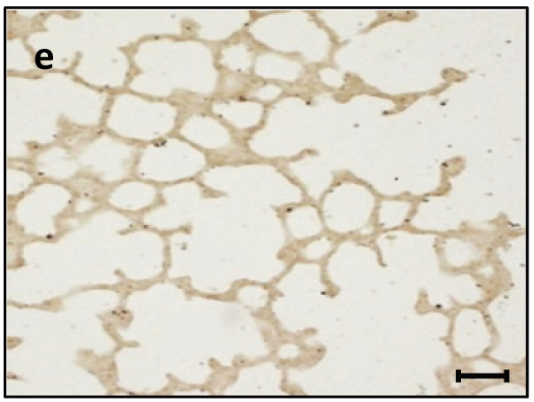

C

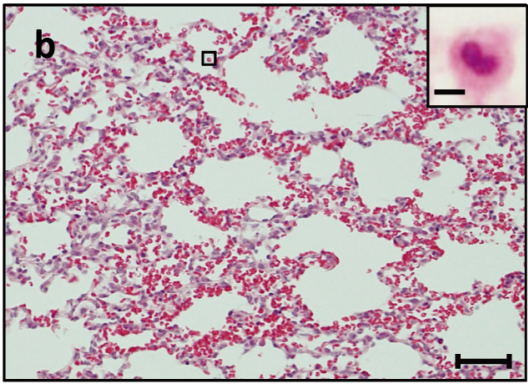

LPS

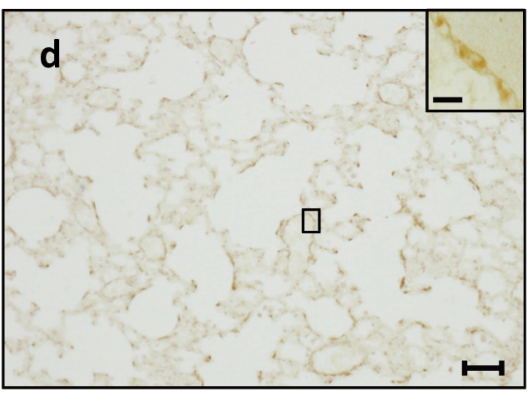

LPS

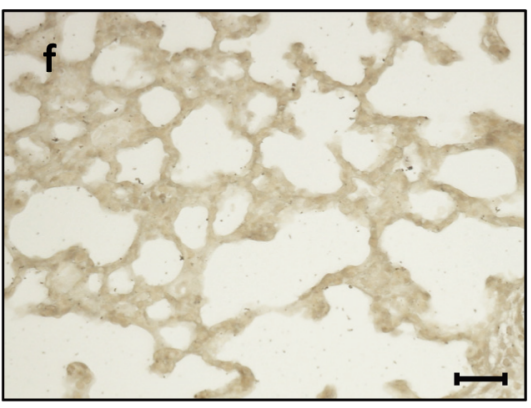

LPS

Fig. 3. Histological analysis of the rat lung treated with or without LPS. (a, b) Representative photographs of HE staining of control (a) and LPS-treated. (b) rats. (c, d) Immunohistochemical analysis of ColVI expression in the lungs of rats treated with LPS (d) and without LPS (c) for $24 \mathrm{~h}$. Scale bars, $50 \mu \mathrm{m}$. The inset in (b) indicates infiltrated neutrophils (scale bar: $5 \mu \mathrm{m}$ ), while that in (d) indicates ColVIpositive interstitial space (scal bar: $4 \mu \mathrm{m}$ ). (e, f) Immunohistochemical analysis of $\alpha$-SMA expression in the lungs of rats treated with LPS (f) and without LPS (e) for $24 \mathrm{~h}$. Scale bars, $30 \mu \mathrm{m}$.

collagen III deficiency, are also involved ${ }^{17}$. Myofibroblasts produce collagens that mainly make up the ECM in pulmonary fibrosis ${ }^{18}$, and so ColVI may have a fundamental role in fibrosis.

Several reports have indicated that tumor-associated macrophages induce ColVI and promote tumor inflammation $^{15}$; however, we did not observe any increase in macrophages under our experimental conditions (Fig. 3b). No induction of ColI proteins and genes was observed after LPS treatment (24 h) (Figs. 1 and 2).

It has been reported that ColVI may be an early phenomenon rather than a late phenomenon in lung fibrosis ${ }^{11}$, and to the best of our knowledge, this is the first report on ColVI activation during early stage systemic inflammation in the rat lung. Activation of ColVI appears to be involved in sepsis-mediated lung fibrosis at an early stage.

Acknowledgments: This work was supported in part by Grants-in-Aid from the Japan Society for the Promotion of Science (24790639 to K. Unuma and 22590629 to T. Aki).

\section{References}

1. Martin C, Papazian L, Payan MJ, Saux P, and Gouin F. Pulmonary fibrosis correlates with outcome in adult respiratory distress syndrome. A study in mechanically ventilated patients. Chest. 107: 196-200. 1995. [Medline] [CrossRef]

2. Fujino N, Kubo H, Suzuki T, He M, Suzuki T, Yamada M, 
Takahashi T, Ota C, and Yamaya M. Administration of a specific inhibitor of neutrophil elastase attenuates pulmonary fibrosis after acute lung injury in mice. Exp Lung Res. 38: 28-36. 2012. [Medline] [CrossRef]

3. Tsuruta Y, Park YJ, Siegal GP, Liu G, and Abraham E. Involvement of vitronectin in lipopolysaccaride-induced acute lung injury. J Immunol. 179: 7079-7086. 2007. [Medline] [CrossRef]

4. Gelse K, Pöschl E, and Aigner T. Collagens-structure, function, and biosynthesis. Adv Drug Deliv Rev. 55: 15311546. 2003. [Medline] [CrossRef]

5. Manabe R, Tsutsui K, Yamada T, Kimura M, Nakano I, Shimono C, Sanzen N, Furutani Y, Fukuda T, Oguri Y, Shimamoto K, Kiyozumi D, Sato Y, Sado Y, Senoo H, Yamashina S, Fukuda S, Kawai J, Sugiura N, Kimata K, Hayashizaki $\mathrm{Y}$, and Sekiguchi K. Transcriptome-based systematic identification of extracellular matrix proteins. Proc Natl Acad Sci USA. 105: 12849-12854. 2008. [Medline] [CrossRef]

6. Bonaldo P, Russo V, Bucciotti F, Doliana R, and Colombatti A. Structural and functional features of the alpha 3 chain indicate a bridging role for chicken collagen VI in connective tissues. Biochemistry. 29: 1245-1254. 1990. [Medline] [CrossRef]

7. Engvall E, Hessle H, and Klier G. Molecular assembly, secretion, and matrix deposition of type VI collagen. J Cell Biol. 102: 703-710. 1986. [Medline] [CrossRef]

8. Keene DR, Engvall E, and Glanville RW. Ultrastructure of type VI collagen in human skin and cartilage suggests an anchoring function for this filamentous network. J Cell Biol. 107: 1995-2006. 1988. [Medline] [CrossRef]

9. Jander R, Rauterberg J, and Glanville RW. Further characterization of the three polypeptide chains of bovine and human short-chain collagen (intima collagen). Eur J Biochem. 133: 39-46. 1983. [Medline] [CrossRef]

10. Malmström J, Tufvesson E, Löfdahl CG, Hansson L, Marko-Varga G, and Westergren-Thorsson G. Activation of platelet-derived growth factor pathway in human asthmatic pulmonary-derived mesenchymal cells. Electrophoresis. 24: 276-285. 2003. [Medline] [CrossRef]
11. Specks U, Nerlich A, Colby TV, Wiest I, and Timpl R. Increased expression of type VI collagen in lung fibrosis. Am J Respir Crit Care Med. 151: 1956-1964. 1995. [Medline] [CrossRef]

12. Unuma K, Aki T, Funakoshi T, Yoshida K, and Uemura $\mathrm{K}$. Cobalt protoporphyrin accelerates TFEB activation and lysosome reformation during LPS-induced septic insults in the rat heart. PLoS ONE. 8: e56526. 2013. [Medline] [CrossRef]

13. Unuma K, Aki T, Matsuda S, Funakoshi T, Yoshida K, and Uemura $\mathrm{K}$. Inducer of heme oxygenase-1 cobalt protoporphyrin accelerates autophagy and suppresses oxidative damages during lipopolysaccharide treatment in rat liver. Hepatol Res. 43: 91-96. 2013. [Medline] [CrossRef]

14. Schnoor M, Cullen P, Lorkowski J, Stolle K, Robenek H, Troyer D, Rauterberg J, and Lorkowski S. Production of type VI collagen by human macrophages: a new dimension in macrophage functional heterogeneity. J Immunol. 180: 5707-5719. 2008. [Medline] [CrossRef]

15. Chen P, Cescon M, and Bonaldo P. Collagen VI in cancer and its biological mechanisms. Trends Mol Med. 19: 410 417. 2013. [Medline] [CrossRef]

16. Naugle JE, Olson ER, Zhang X, Mase SE, Pilati CF, Maron MB, Folkesson HG, Horne WI, Doane KJ, and Meszaros JG. Type VI collagen induces cardiac myofibroblast differentiation: implications for postinfarction remodeling. Am J Physiol Heart Circ Physiol. 290: H323-H330. 2006. [Medline] [CrossRef]

17. Volk SW, Wang Y, Mauldin EA, Liechty KW, and Adams SL. Diminished type III collagen promotes myofibroblast differentiation and increases scar deposition in cutaneous wound healing. Cells Tissues Organs. 194: 25-37. 2011. [Medline] [CrossRef]

18. Zhang K, Rekhter MD, Gordon D, and Phan SH. Myofibroblasts and their role in lung collagen gene expression during pulmonary fibrosis. A combined immunohistochemical and in situ hybridization study. Am J Pathol. 145: 114-125. 1994. [Medline] 